\title{
APPLICATION AND VALIDATION OF A DIRECTIONAL SOLIDIFICATION MODEL AND DENDRITE MORPHOLOGY CRITERION FOR COMPLEX, SINGLE-CRYSTAL CASTINGS
}

\author{
J.D. Miller, K.J. Chaput, D.S. Lee, and M.D. Uchic \\ Materials and Manufacturing Directorate, Air Force Research Laboratory \\ 2230 Tenth St, Wright Patterson AFB, OH 45433
}

Keywords: directional solidification, process modeling, dendrite growth

\begin{abstract}
Extensive work is required to determine optimal processing conditions (e.g. withdrawal, rate furnace temperature) for directional solidification of single crystal castings in the complex geometries required for modern flight hardware. Prior work used fundamental solidification theory and modeling to adapt classical defect-formation criteria to realistic non-planar solidification and develop a geometric-independent metric for preferred casting conditions. The continuation of this work consists of applying and validating these learned metrics to representative blade geometries. The overarching objective is to develop an integrated set of modeling tools capable of linking process parameters and alloy properties to modern design methods through the prediction of critical microstructural features: dendritic structure, defect formation, and porosity. Thermocouple measurements from directional solidification experiments and full-cross-section metallographic characterization of resulting castings have been compared to model predictions and show good agreement. New parameters from solidification models have been developed and demonstrated for the onset and extent of dendrite morphology changes in a turbine blade geometry.
\end{abstract}

\section{Introduction}

The process for fabricating single crystal blades and vanes has remained relatively unchanged for more than 40 years [1]. The advancements in geometric complexity and alloying have contributed to more capable single-crystal airfoils in modern turbine engine hardware [2]. Future single-crystal hardware has been envisioned with thinner walls, more internal cooling capability, and better blade-surface cooling efficiency. The means to produce such hardware will rely on a more fundamental understanding of the directional solidification process, since the complexity of the geometry results in more complex thermal fields during solidification and the further reduced wall thickness approaches the scale of a single dendrite across the wall. These geometric requirements also merit the evaluation of unconventional directional solidification processes and will require the use of process modeling tools to assist in process design in lieu of historical experience.

One such unconventional directional solidification process is the Liquid Metal Cooling (LMC) process [3-4]. The LMC process utilizes a liquid metal coolant in the cold region of a directional solidification furnace which increases heat extraction thus increasing the thermal gradient in the transition region, resulting in increased cooling rate, refined microstructure scale and reduced defect propensity [5-8]. A floating baffle of ceramic beads typically insulates the liquid metal coolant from the hot zone of the DS furnace [5,7-9]. This process has been studied intermittently at the research scale over the past two decades [517], with a primary application of ground-based turbine applications. The increased heat extraction provided by the LMC process is primarily achieved through enhanced lateral cooling, thus increasing the solidification-front curvature.

The non-axial thermal fields during the LMC process increase the propensity for lateral growth - the preferential growth of secondary arms overgrowing well-aligned, neighboring primary dendrite arms. Increased solidification-front curvature, or more explicitly, increased inclination of the solid-liquid interface has also been shown to lead to grain nucleation due to undercooling ahead of the solid-liquid interface at the mold wall where inclination of the solid-liquid interface is typically highest [1620]. The mechanism of lateral growth and its relationship to solid-liquid interface inclination angle has been studied through coupled casting trials and solidification modeling to develop new criteria and thresholds for changes in dendrite growth morphology (Figure 1) [16-17,21]. This work was conducted on simple geometries but has not been demonstrated for representative blade geometries.

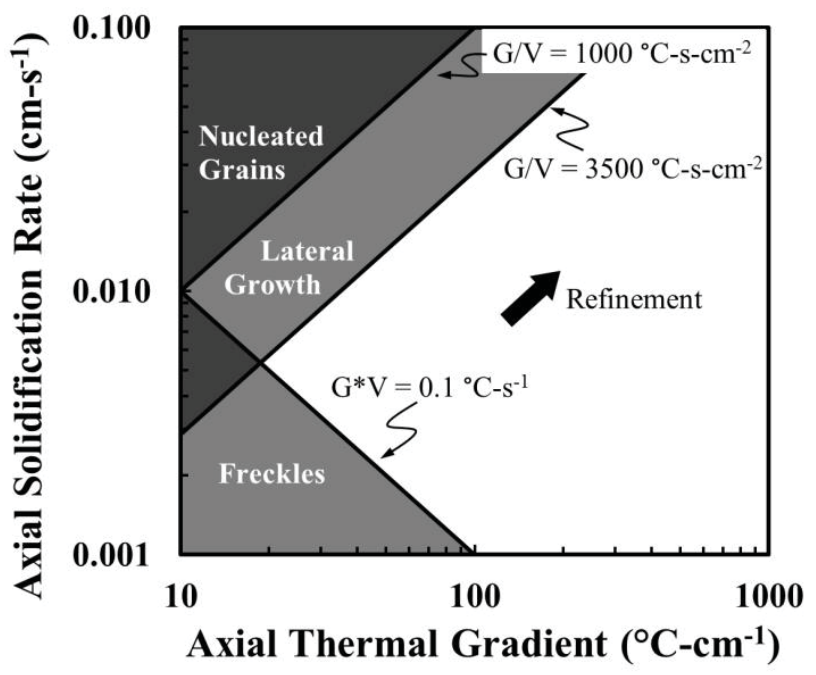

Figure 1. Classical defect formation map with superimposed regions of axial growth, lateral growth and grain nucleation [16].

Solidification modeling of the directional solidification process has been used extensively in the literature to support experimental evidence for microstructure scale and dendrite morphologies $[5,8,11,14-26]$. This research primarily included finite-element fluid flow and heat transfer modeling using commercially available software tools or analogous modeling frameworks. Generally, these investigations were conducted on relatively simple casting geometries such as bars and slabs. One 
particular set of investigations (1) developed a criterion for the formation of different dendrite growth morphologies in both Bridgman and Liquid Metal Cooling (LMC) directional solidification processes and (2) applied fundamental solidification theory to thermocoupled casting trials and validated solidification models $[8,14-17]$. These investigations showed that for these simple geometries the model could accurately predict the influence of non-axial thermal fields to dendrite scale and dendrite growth morphologies in both Bridgman and Liquid Metal Cooling (LMC) processed material. However, these criteria have not been used in relevant blade geometries in which geometric complexity could play a significant role in the evolution of the dendritic microstructure.

In this manuscript, a process model has been employed to predict local thermal fields and first-order microstructural features in actual turbine hardware and will be demonstrated through comparison to cooling curves from thermocoupled experimental casting trials of both the Bridgman and LMC processes, as well as ex-situ serial sectioning of turbine blades for microstructural evaluation. Associated parametric analyses were also conducted to identify the influence of key process variables on microstructure-controlling thermal field parameters.

\section{Methodology}

Representative turbine blades using a production relevant mold configuration with alloy René N4 were cast using an ALD vacuum furnace. The specific details of the furnace have been published elsewhere [5]. The ALD furnace has the unique ability to operate in both a Bridgman and Liquid Metal Cooling (LMC) mode, which affords a spectrum of thermal conditions to be evaluated during this study. Four casting conditions were evaluated: (1) a baseline Bridgman process with a conventional withdrawal rate, (2) a baseline LMC process with a typical LMC withdrawal rate, (3) an increased withdrawal rate LMC trial, and (4) a further increased withdrawal rate LMC trial. The accelerated withdrawal rates were selected to provide castings with lateral growth and nucleated grains, respectively, according to research from simple castings [16-17] and were set to 2, 3 and 5 times the baseline Bridgman withdrawal rate for the LMC casting trials. Further details of the casting conditions cannot be disclosed due to the sensitivity of these parameters for representative geometries. Type $\mathrm{C}$ thermocouples were embedded in the casting mold at the mid-span and root of the airfoil (Figure 2).

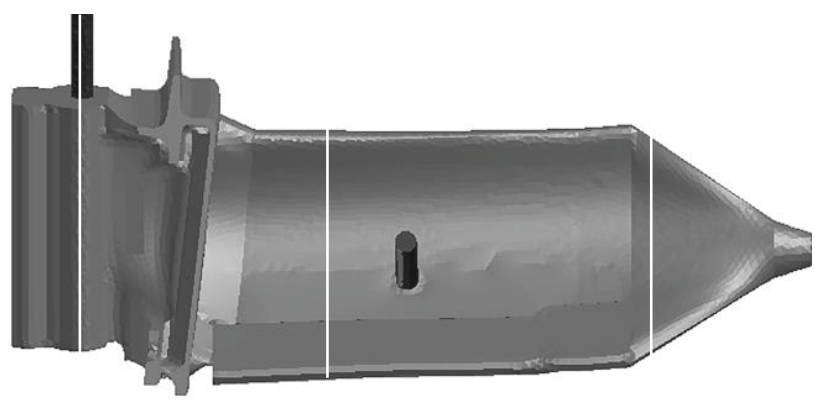

Figure 2. CAD model of the casting geometry depicting the location of transverse cross sections indicated by white lines and position of thermocouples indicated as black cylinders.
The castings were sectioned transverse to the growth direction at three locations - the ramp below the representative blade, the mid-span of the airfoil and the root (Figure 2). These crosssections were subsequently characterized using Backscatter Electron Imaging (BEI) to reveal the underlying dendrite morphology. Custom montage automation scripts were developed to collect BEI data from the entire cross-section using a Tescan Lyra FIB-SEM at a resolution of $1 \mu \mathrm{m}$. These scripts include the ability to optimize both focus and brightness/contrast to maintain consistent imaging conditions across the $\mathrm{cm}$-scale surfaces, for total acquisition times that exceed 24 hours as the montages were comprised of up to 2000 tiles. Post-processing of the montage tile data was performed using both custom Python scripts and a tile stitching algorithm included in the image processing software FIJI [27]. Using the cross-section BEI data, the local dendrite morphology was categorized into one of three growth modes: axial growth, lateral growth, or nucleated grains. A cross-section was characterized lateral growth if at least $1 \mathrm{~cm}^{2}$ of the cross section exhibited lateral growth, and nucleated grains if any nucleated grains were observed within the cross-section. Otherwise the section was identified as axial growth.

The directional solidification processes were simulated using the finite-element, solidification tool ProCAST ${ }^{\mathrm{TM}}$ [ProCAST is a trademark of ESI Group, Inc.]. The nominal modeling parameters and boundary conditions used in these models have been described in previous work [14,28]. One-to-one simulations of experimental casting trials were modeled. Predicted cooling profiles of the mold, from regions surrounding the location of the thermocouples, at both the exterior of the mold and the mold-metal interface were compared to the experimental results.

Previously developed and demonstrated defect formation criteria were calculated from each of the solidification models for the formation of such defects in complex mold configurations [1417]. Specifically, these defect formation criteria are (1) $G_{\text {axial }} V_{\text {axial }}{ }^{-1}$ and (2) solid-liquid-interface inclination angle. $G_{\text {axial }}$ and $V_{\text {axial }}$ are the thermal gradient and dendrite-growth velocity parallel to the withdrawal direction calculated at 0.4 fraction solid according to equations (1) and (2) in which $G$ is the total thermal gradient, $\theta$ is the inclination angle and $V$ is the isotherm velocity. The solid-liquid interface inclination angle, referred to as simply inclination angle in this manuscript, is the angle between the normal of the solid-liquid interface and the withdrawal direction at the same fraction solid. The different metrics were mapped and compared to the dendrite-morphology classifications from the experimental cross-sections to evaluate effectiveness at predicting defect formation in these complex geometries.

$$
\begin{aligned}
& G_{\text {axial }}=G \cos (\theta) \\
& V_{\text {axial }}=V / \cos (\theta)
\end{aligned}
$$

In addition, a simulated parametric analysis was conducted to evaluate the effect of specific process variables on the resulting local thermal conditions - thermal gradient, cooling rate, axial growth velocity and inclination angle. Withdrawal rate and furnace temperature were the process variables investigated for a research-scale furnace for both the Bridgman and LMC processes. The withdrawal rate was evaluated between 
approximately 0.2 times to 2.0 times the baseline withdrawal rate for each of the Bridgman and LMC processes, totaling 8 different set points for withdrawal rate sensitivity for each process. The furnace temperature was parametrically varied by $50{ }^{\circ} \mathrm{C}$ around the baseline furnace temperature. Simulations representative of a Bridgman production-scale furnace and casting configuration were also evaluated and compared to process-parameter equivalent research-scale casting simulations for a quantitative comparison of the effect of furnace scale. For all analyses, normalized differences from nodes in the mid-span of the airfoil were directly compared to obtain relative importance of the input parameters. In all cases, the values were normalized by the baseline input parameters and predictions from the baseline simulations of the Bridgman and LMC processes.

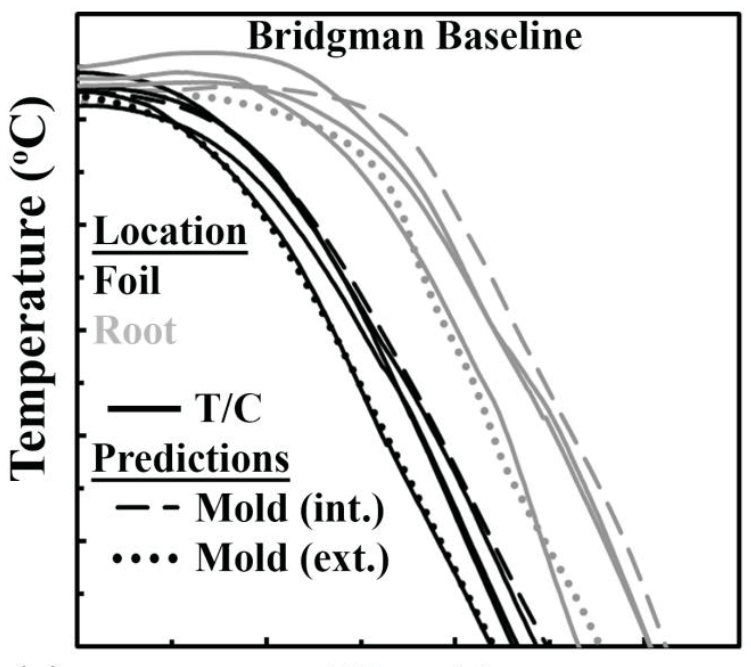

(a)

Time (s)

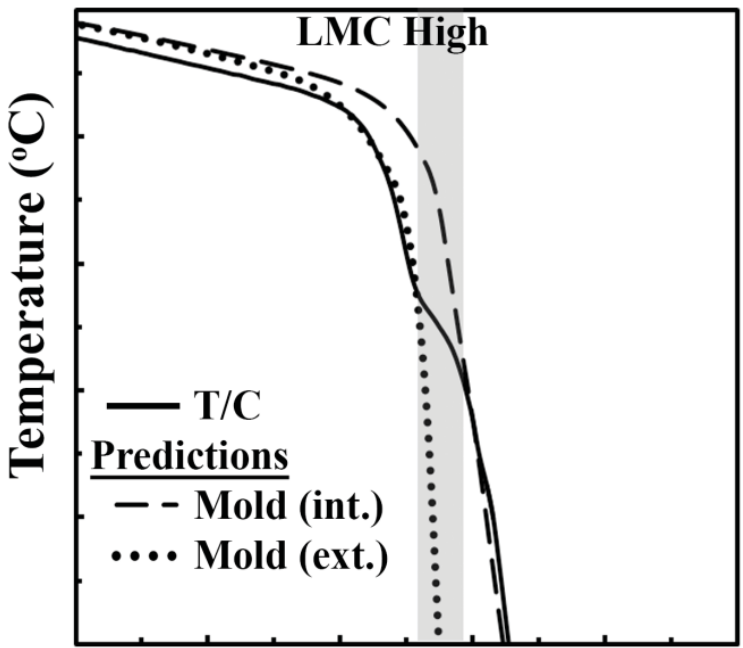

(c)

Time (s)
Results

Research-Scale Turbine Blade Castings: Measured and Predicted Thermal Response

Castings of all of the conditions evaluated in this study were successfully produced with thermocouples that survived the casting process allowing for measurement of the thermal profile to validate the casting model. For both the Bridgman and LMC baseline conditions, multiple thermocouples survived from multiple castings allowing further evaluation of cooling variability and sensitivity to slight deviations in thermocouple position. To validate the model, the cooling rates of nodes at three locations - near the exterior of the mold surface, in the alumina sheathing that penetrates the mold, and at the moldmetal interface were plotted in comparison to the measure temperature profile (Figure $3 \mathrm{a}$ and $\mathrm{b}$ ) for both the LMC and Bridgman castings.
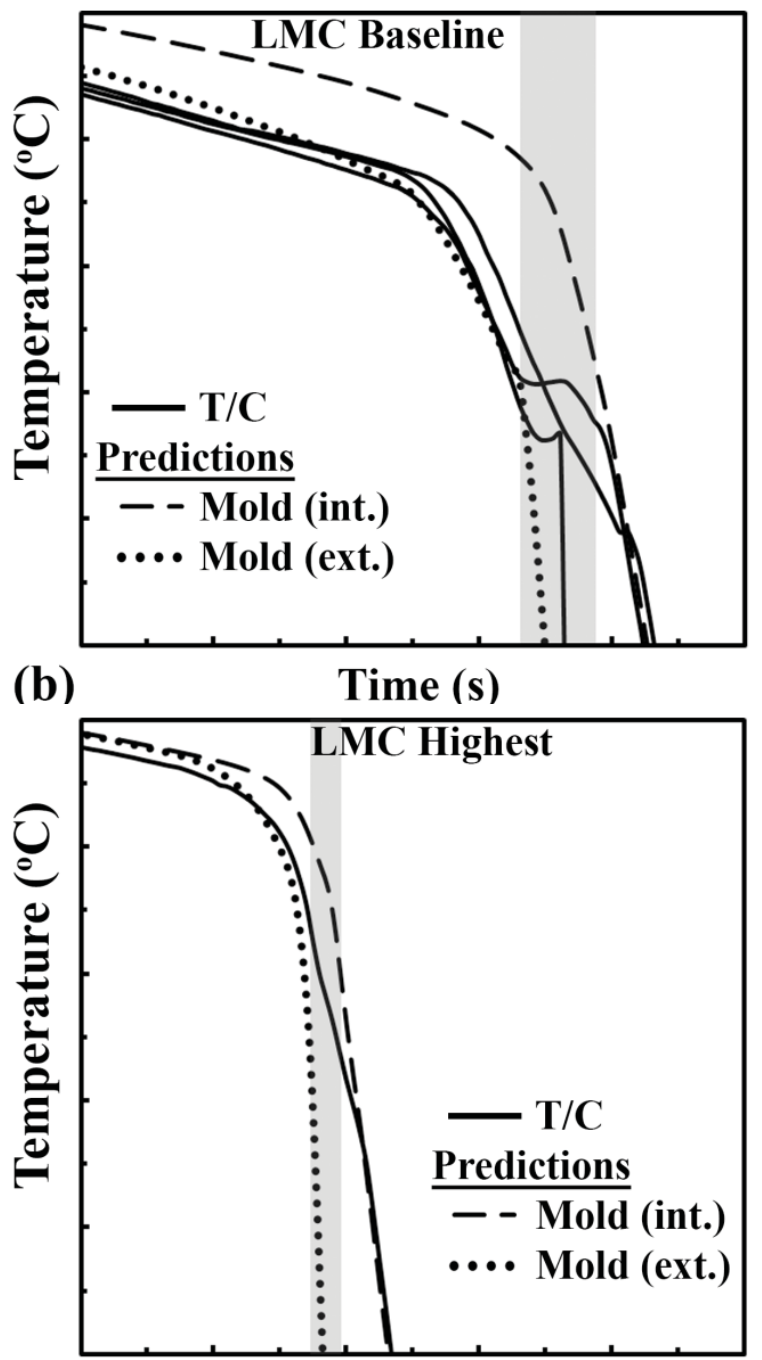

(d)

Time (s)

Figure 3. Comparison of predicted temperature profiles and thermocouple cooling curves: (a) the Bridgman process in which different regions in the simulation near the thermocouple junction are represented with different line styles while the different colors (gray and black) represent different thermocouple locations in the casting, and the LMC process at different withdrawal rates (b-d) in which the different colors correspond to different withdrawal rates and the different line styles correspond to experimental (solid) and predicted cooling profiles for the mold at the exterior surface (dotted) and metal surface (dashed) near the thermocouple location. The gray bands in (b-d) denote the time range over which the thermocouple data for each particular withdrawal rate passed through the floating baffle. 
For the Bridgman process, predicted cooling rates of nodes near the exterior mold surface at the position of the thermocouples were within $0.02^{\circ} \mathrm{C}-\mathrm{s}^{-1}$ at the solidus compared to the respective thermocouple data for all casting conditions (Figure 3). It is believed that this is due to the thermocouples crossing (shorting) at the mold surface due to physical limitations for the placement of thermocouples in the ALD furnace setup. The time temperature history of the predicted cooling curves and thermocouple data were consistent from the start of withdrawal to temperatures far below the solidification temperature.

For all LMC casting trials, the thermocouple temperature profile coincided with the time-temperature profile of the exterior mold location, just as in the Bridgman trial, until the thermocouple location lowered to within the floating baffle in the transition region of the furnace. At this point the thermocouple data transitioned and subsequently coincided with the temperature profile of the mold-metal interface (Figure 3b). It was concluded that the molten bath separated the previously incontact (shorted) thermocouple wires near the exterior mold surface through mold cracking and wire buoyancy allowing for temperature readings at the mold-metal interface after immersion in the ceramic baffle. These observations were consistent across a range of withdrawal rates and scaled with withdrawal distance and thickness of the floating baffle (Figure $3 b)$.

\section{Research-Scale Turbine Blade Castings:}

Observed and Predicted Dendrite Morphology

Transverse metallographic sections from the castings exhibited regions of differing dendrite morphologies - axial growth, lateral growth and nucleated grains - as designed (Figure 4). Axial growth throughout the entire blade cross section was observed in 7 of the 12 sections evaluated. Typical axial growth for the Bridgman and LMC processes was observed (Figure 4a and $4 \mathrm{~b}$, respectively), showing significant dendrite refinement with the LMC process, even for complex casting configurations. Lateral growth was observed at the highest withdrawal rate within the mid-span of the airfoil (Figure 4c). The particular section in Figure 4c was imaged at the middle of a rib at the boundary of lateral growth (above in the figure) and axial growth (below in the figure). The presence of nucleated grains was observed in the root section of the two highest withdrawal rate LMC castings. The observed dendrite morphologies have been summarized (Table I).
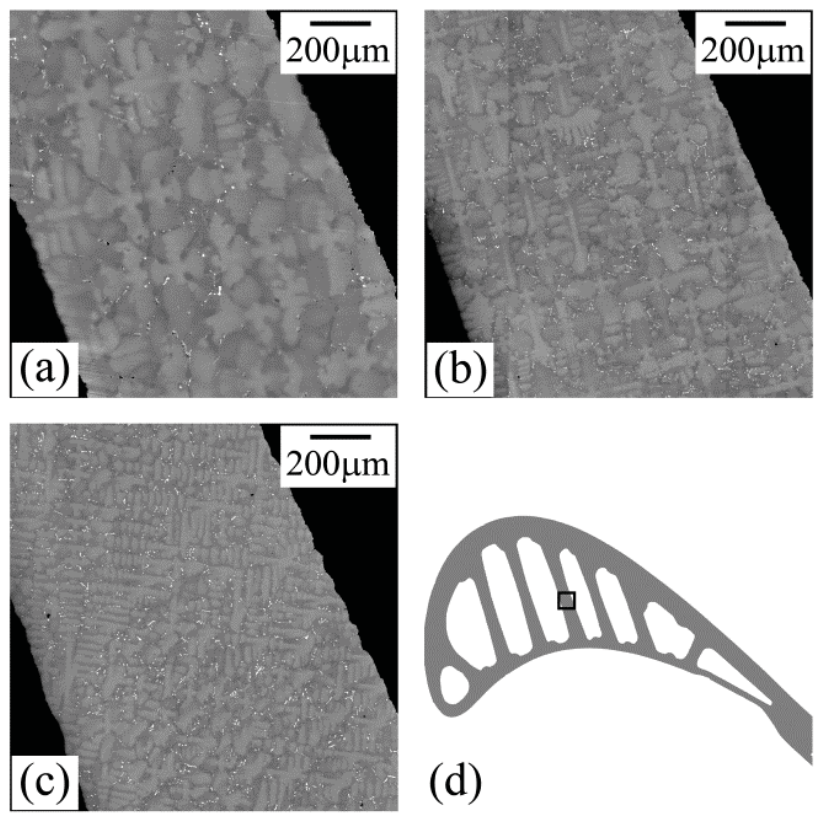

(d)

Figure 4. Transverse micrographs at the mid-span of the airfoil across a rib imaged by BEI of (a) baseline Bridgman, (b) high withdrawal rate LMC and (c) highest withdrawal rate LMC. Micrographs were imaged in the location as indicated by the outlined window in (d).

Metallographic sections of dendrite morphology and maps of defect formation criteria compare favorably both with respect to qualitative shape and quantitatively self-consistent for the onset of lateral growth and nucleated grains (Figure 5). At the midspan of the airfoil, for example, contour plots of inclination angle and " $\mathrm{G}_{\text {axial }} \mathrm{V}_{\text {axial }}{ }^{-1}$ " predicted the onset of lateral growth (Figure 5). Lateral growth was observed in this slice of the blade on the suction side of the blade near the leading edge (Figure 5a). Lateral growth continues towards the suction side midway down the ribs and stops at the superimposed black line (Figure 5a). The secondary growth direction in which lateral growth occurred varied among the four possible in-plane $<001>$ crystallographic directions throughout the cross-section of the blade.

Table I. Observed dendrite morphology for each cross-section of each casting trial. "A", "L" and "N" designate complete axial growth, lateral growth, and nucleated grains, respectively.

\begin{tabular}{ccccc}
\hline Section & $\begin{array}{c}\text { Bridgman } \\
\text { Baseline }\end{array}$ & $\begin{array}{c}\text { LMC } \\
\text { Baseline }\end{array}$ & $\begin{array}{c}\text { LMC } \\
\text { High WDR }\end{array}$ & $\begin{array}{c}\text { LMC } \\
\text { Highest WDR }\end{array}$ \\
\hline Ramp & A & A & A & A \\
Foil & A & A & A & L \\
Root & L & L & N & N \\
\hline
\end{tabular}




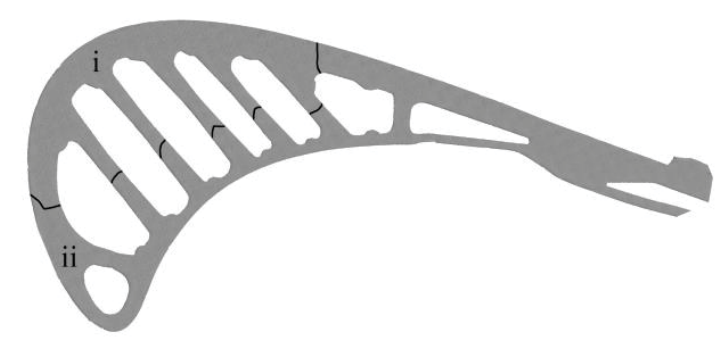

(a)

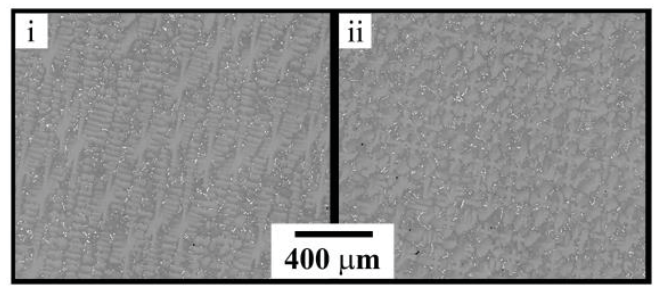

(b)

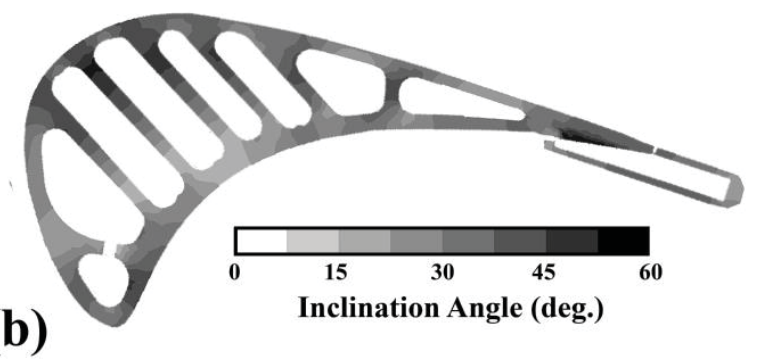

(c)

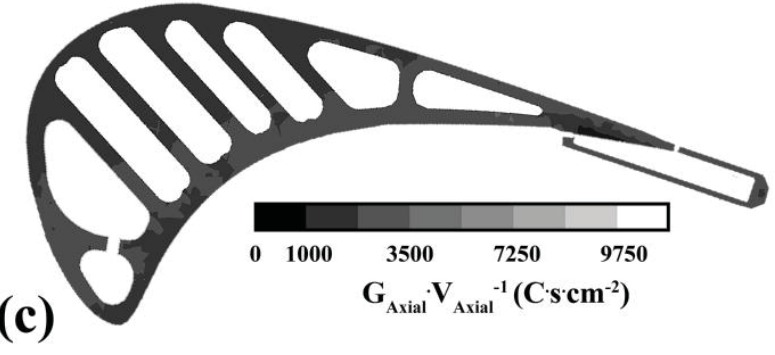

Figure 5. (a) BEI montage of the mid-span of a representative blade casting with superimposed black line for the boundary between lateral growth and axial growth. Predicted grayscale contour maps of (b) inclination angle and (c) classical defect formation criteria $\left(G_{\text {axial }} \cdot V_{\text {axial }}{ }^{-1}\right)$ for the same cross-section as in (a).

Due to the brevity of this manuscript, it can only be stated that the results from the remainder of the cross sections were selfconsistent with those presented in Figure 5 with one exception: the classical defect formation criteria over-predicted the onset of lateral growth in the ramp section of the Bridgman process (Table II). Quantitatively, axial growth occurred with an inclination angle up to $30^{\circ}$. The onset of lateral growth was predicted at an inclination angle of $30^{\circ}$, and the onset of nucleated grains was observed at an inclination angle of $66^{\circ}$. The threshold for the onset of nucleated grains according to this research may be as low as $53^{\circ}$ due to the lack of data between $53^{\circ}$ and $66^{\circ}$. Likewise, based on predictions of classical defect formation criteria, axial growth occurred down to $1800^{\circ} \mathrm{C}-\mathrm{s}-\mathrm{cm}^{-}$ ${ }^{2}$, except for the ramp section of the Bridgman process. The onset of lateral growth was observed between 1400 to $500{ }^{\circ} \mathrm{C}$-s$\mathrm{cm}^{-2}$. The onset of nucleated grains was observed at $250{ }^{\circ} \mathrm{C}-\mathrm{s}-$ $\mathrm{cm}^{-2}$ and below.
Table II. Maximum inclination angle and minimum " $\mathrm{G}_{\text {axial }} \mathrm{V}_{\text {axial }}$ 1 " at 3 locations designated "ramp", "airfoil" and "root" from 4 different casting simulations representative of the four casting trials produced via the Bridgman and LMC processes.

\begin{tabular}{|c|c|c|c|c|c|}
\hline $\begin{array}{c}\text { Thermal } \\
\text { Field }\end{array}$ & Section & $\begin{array}{c}\text { Bridgman } \\
\text { Baseline }\end{array}$ & $\begin{array}{c}\text { LMC } \\
\text { Baseline }\end{array}$ & $\begin{array}{l}\text { LMC } \\
\text { High } \\
\text { WDR }\end{array}$ & $\begin{array}{c}\text { LMC } \\
\text { Highest } \\
\text { WDR }\end{array}$ \\
\hline \multirow{3}{*}{$\begin{array}{c}\text { Inclination } \\
\text { Angle } \\
\text { (deg.) }\end{array}$} & Ramp & 22 & 25 & 26 & 29 \\
\hline & Airfoil & 14 & 14 & 30 & 53 \\
\hline & Root & 40 & 30 & 66 & 84 \\
\hline \multirow{3}{*}{$\begin{array}{c}\text { Gaxial } \\
\text { Vaxial } \\
\left({ }^{\circ} \mathrm{C}-\mathrm{s} / \mathrm{cm}^{2}\right)\end{array}$} & Ramp & 625 & 2750 & 2500 & 1800 \\
\hline & Airfoil & 5000 & 5100 & 3000 & 1400 \\
\hline & Root & 500 & 1000 & 250 & 20 \\
\hline
\end{tabular}

Parametric Analysis of Directional Solidification

A parametric analysis of the Bridgman and LMC processes at the research scale indicated that the predicted thermal-field parameters are more sensitive to changes in withdrawal rate than changes in furnace temperature (Figures 6). In both cases, the axial thermal gradient maximized at an intermediate withdrawal rate, although the relative difference was comparatively minor across a broad range of withdrawal rates (Figure 6a). The relative difference in axial growth velocity was nearly one-toone with the relative difference in withdrawal rate (Figure 6a). Inclination angle increased approximately one-to-one with increasing withdrawal rate, while classical defect formation criteria decreased by approximately one-to-one, corresponding to increased likelihood for nucleated grains, with increasing withdrawal rate (Figure 6b). Comparison of Bridgman versus LMC parametric results demonstrate that the thermal gradient, and inclination angle were more sensitive for the LMC process, and classical defect formation criteria were more sensitive for the Bridgman process (Figure 6b).

Across a practically achievable range of furnace temperature and at the same baseline withdrawal rate, the axial thermal gradient increased with increasing furnace temperature by $19 \%$ across a $50{ }^{\circ} \mathrm{C}$ temperature increase, but the axial growth velocity decreased by $5 \%$ with increasing furnace temperature across the same temperature difference. These differences are insignificant to the onset of lateral growth or grain nucleation, as the inclination angle only differed by $7 \%$ and classical defect formation criteria by $24 \%$, for a logarithmic relationship. 

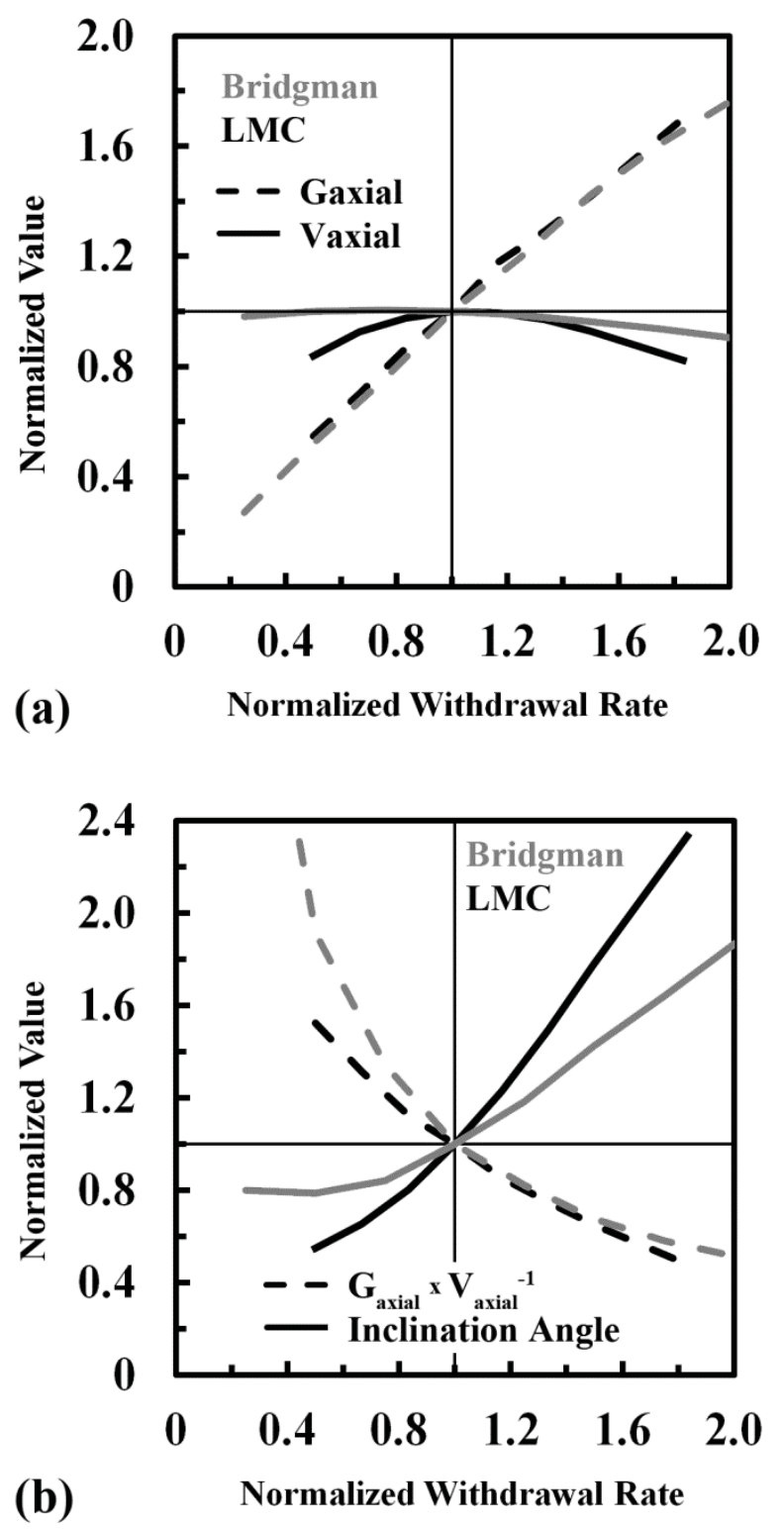

Figure 6. Effect of varying the the withdrawal rate from the baseline withdrawal rate on: (a) axial thermal gradient and axial dendrite growth velocity, and (b) inclination angle and " $\mathrm{G}_{\text {axial }} \mathrm{V}_{\text {axial }}{ }^{-1}$ ".

Comparison of model predictions for research scale versus production scale configurations for the Bridgman directional solidification process show significant degradation in thermal performance of the process for production scale. A node-bynode comparison of multiple thermal parameters between production-scale and research-scale models was conducted within a $1 \mathrm{~cm}$ axial band in the mid-span of the airfoil. In this band, the average predicted temperature prior to withdrawal lowered $34^{\circ} \mathrm{C}$ for the production scale as compared to the research scale (Figure 7). In a similar analysis, production-scale predictions displayed an average degradation of $35 \%$ for the axial thermal gradient and an $18 \%$ increase in axial growth velocity, resulting in a $43 \%$ reduction in the classical defect formation criteria. The inclination angle showed an average decrease of 5.2 degrees. For context, the average inclination angle of the research configuration was $12.1 \pm 7.6 \mathrm{deg}$.

(a)

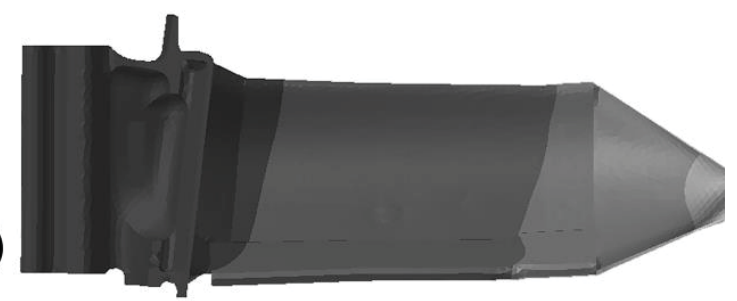

(b)

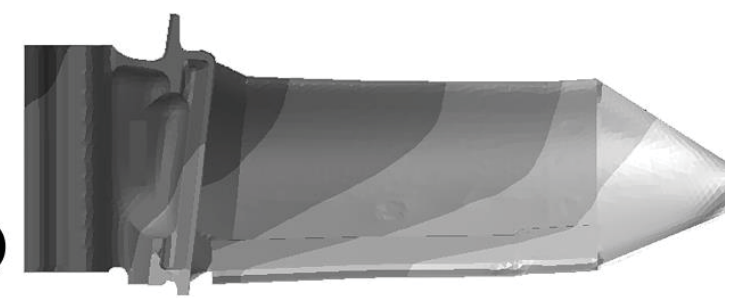

Figure 7. Thermal profiles at the start of withdrawal for the (a) research scale and (b) production scale configurations of the Bridgman directional solidification process. Each grayscale band represents a temperature change of $10^{\circ} \mathrm{C}$.

\section{Discussion}

Non-Axial Thermal Fields

Dendrite morphology predictions from this study demonstrate the importance of accounting for non-axial thermal fields. Due to the immaturity of the analytical tools and a lack in the sophistication of experiments during the early development of classical defect formation criteria, it was assumed that the solidliquid interface was flat. Apparent by this study and previous ones on simpler geometries [8,16-17,29], the assumption of a flat solid-liquid interface is invalid for even relatively simple geometries. By accounting for the inclined solid-liquid interface, model predictions can lead to a drastic difference between the thermal-parameter magnitudes previously assumed and those thermal parameters relevant to solidification behavior.

\section{Prediction of Dendrite Morphology}

The ability of the defect formation criteria to predict the onset and extent of differing dendrite morphologies for representative blade geometries was evaluated in this work. The quantitative value for the onset of lateral growth, a $30^{\circ}$ inclination from this work, was approximately consistent with previous results on simple castings, specifically $25^{\circ}$, while the onset of nucleated grains, $66^{\circ}$ from this work, differed from previous work's threshold of $45^{\circ}$ [16-17]. The predicted thresholds for lateral growth and nucleated grains using classical defect formation criteria were less consistent with prior results of 3500 and 1000 ${ }^{\circ} \mathrm{C}-\mathrm{s}-\mathrm{cm}^{-2}$, respectively. More work is required to completely demonstrate quantitative thresholds for these defect formation criteria, especially for geometries in which the casting surfaces are not parallel to the withdrawal direction, such as ramps. Specifically, Figure 5b showed three regions that should have resulted in lateral growth due to inclination angle greater than 30 but only one of these regions exhibited lateral growth. In these cases additional rules may be required to properly account for dendrite morphology, such as accounting for alignment of secondary growth directions relative to the inclined 
solidification front. However, this work demonstrates the utility of the proposed parameters to semi-quantitatively predict the onset of both lateral growth and nucleated grains.

Table II shows that inclination angle is more effective than " $\mathrm{G}_{\text {axial }} \mathrm{V}_{\text {axial }}{ }^{-1 "}$ " for the prediction of lateral growth, which is logical since the competition between primary dendrite tips and secondary dendrite tips is primarily governed by their relative growth velocities, which is closely related to inclination angle. In addition, the inclination angle criterion accurately predicted the presence of nucleated grains. This finding may correspond to a special case of grain nucleation that is preceded by a highly inclined solid-liquid interface that enhances undercooling ahead of the dendrite tips at the wall. It is postulated that the inclination angle criteria will be inadequate at predicting nucleated grains for conditions with poor thermal gradient with a relatively flat solid-liquid interface.

\section{Parametric Analysis}

The parametric analysis conducted in this work identified key attributes of the Bridgman process that have been summarized in this section. Comparison of the Bridgman and LMC processes from previous work $[8,14]$ indicated that the axial thermal gradient at the solid-liquid interface during the LMC process was more sensitive to withdrawal rate than the Bridgman process. From a different perspective, the axial thermal gradient during the Bridgman process appeared to be more robust, that is insensitive to small changes in process parameters, than the LMC process. The same result is observed in this work for complex geometries.

The furnace temperature parametric analysis showed that the thermal gradient increased by approximately $19 \%$ while the axial growth velocity decreased by approximately $5 \%$ for the same increase in furnace temperature. The interplay of these parameters resulted in effectively no benefit for increasing the furnace temperature, which is counter-intuitive to the concept of increasing the macroscopic thermal gradient. These predictions were from simulations at the same withdrawal rate, so the influence of the position in the solid-liquid interface may affect the relative thermal gradient. Further research is required to determine whether improvements to the thermal efficiency can be achieved when raising the furnace temperature by tailoring the withdrawal rate to each furnace temperature independently.

\section{$\underline{\text { Research and Production Scale Comparisons }}$}

As described previously, relatively large reductions of thermal efficiency were observed when upscaling casting configurations from the research scale to the production scale. Further evaluation of the casting model showed that the origin of the degradation could be related to two factors, both related to a reduced radiation heat transfer efficiency at larger length scales. First, and most logical, the radiation cooling was less effective at the production scale, especially for those blade locations inside the cluster with poor view factors to the cold furnace walls. Second, and less intuitive, the initial thermal profile prior to withdrawal for the production scale was broadly colder than the research scale analog due to reduced heating efficiency during the soak (Figure 7). Thus, a lower macroscopic thermal gradient existed in the furnace based on a lower peak temperature in regions of the casting
The thermal field comparisons between the two different configuration scales showed degradation of the thermal efficiency, but the impact of this degradation is more significant than assessment of thermal field alone. The degradation of thermal parameters amounts to an increase in microstructure scale of $15 \%$ and $8 \%$ for PDAS and SDAS, respectively based on the physics-based models for PDAS and SDAS [30-31]. In addition, the likelihood to form nucleated grains increases as shown by the decrease in the classical defect criteria $\left(\mathrm{G}_{\text {axial }} \mathrm{V}_{\text {axial }}\right.$ $\left.{ }^{1}\right)$ and drop in inclination angle. While the drop in inclination angle may not appear signification by evaluating the distribution of the inclination angle reported for the research configuration, the increase in the mean would lead to a rather significant increase in the percentage of nodes that would predict lateral growth or a nucleated grain.

These results highlight the reality that results from researchscale casting trials should be used carefully when assessing the value of emerging processes, the tuning of process parameters in simple geometries, or the optimization of a directional solidification process for a given geometry. While the research scale can be treated as best-case and provides some insights on solidification behavior, the difference in thermal parameters may require alterations to the furnace temperature and withdrawal rates to create production-scale "equivalent" castings.

\section{Conclusions}

Experimental blade-representative castings were successfully produced for a range of casting conditions including both Bridgman and LMC modes at a range of withdrawal rates.

- Temperature profiles were successfully obtained for nearmetal cooling profiles in representative blade geometries.

- A methodology to montage large cross-sections of dendrite morphology via BEI was demonstrated.

- A range of intended dendrite morphologies were successfully produced within a representative blade geometry from designed process conditions

A solidification model has been validated for bladerepresentative hardware and is capable of predicting both temperature profiles and dendrite morphologies.

- The predicted temperature profiles were quantitatively accurate compared to the thermocouple measurements.

- Geometry-independent morphology criteria (1) were demonstrated for complex geometries, (2) account for solidification front curvature and (3) reduce to classic defect formation criteria for simpler geometries or for the case of a flat solid-liquid interface.

- Developed or applied maps of defect formation criteria coincide with regions of differing dendrite morphology observed in representative blade geometries.

A parametric analysis demonstrated the relative importance of withdrawal rate, furnace temperature and configuration scale.

- Withdrawal rate affected thermal-field parameters more significantly than furnace temperature for the range of conditions evaluated.

- The change in configuration scale from research to production dramatically degraded the thermal efficiency of the Bridgman process. 


\section{References}

1. M. McLean, Directionally Solidified Materials for High Temperature Service, London: The Metal Society, 1983.

2. R.C. Reed, The Superalloys: Fundamentals and Applications, Cambridge University Press, 2006.

3. J.G. Tschinkel, A.F. Giamei and B.H. Kearn, "Apparatus for Casting of Directionally Solidified Articles," (U.S. Patent No 3,763,926), 1973.

4. A.F. Giamei and J.G Tschinkel, Metall and Mater Trans A, 7 (1976) 1427-1434.

5. A.J. Elliott, S. Tin, W.T. King, S.-C. Huang, M.F.X. Gigliotti and T.M. Pollock, Metall and Mater Trans A, 35 (2004) 3221 3231 .

6. J. Zhang and L.H. Lou, J Mater Sci Technol, 23 (2007) 289300 .

7. C.L. Brundidge, J.D. Miller and T.M. Pollock, Metall and Mater Trans A, 42 (2011) 2723-2732.

8. J.D. Miller and T.M. Pollock, Metall and Mater Trans A, 43 (2012) 2414-2425.

9. A.J. Elliott, G.B. Karney, M.F.X. Gigliotti and T.M. Pollock, Superalloys 2004, eds. K.A. Green, T.M. Pollock, H. Harada, T.E. Howson, R.C. Reed, J.J. Schirra and S. Walston (The Minerals, Metals \& Materials Society, 2004) 421-430.

10. M. Konter, E. Kats and N. Hofmann, Superalloys 2000, eds. T.M. Pollock, R.D. Kissinger, R.R. Bowman, K.A. Green, M. McLean, S. Olson and J.J. Schirra (The Mineral, Metals \& Materials Society, 2000) 189-200.

11. T.J. Fitzgerald and R.F. Singer, Metall Trans A, 28 (1997) $1377-1383$

12. A. Lohmüller, W. Esser, J. Grossman, M. Hördler, J. Preuhs and R.F. Singer, Superalloys 2000, ed. T.M. Pollock, R.D. Kissinger, R.R. Bowman, K.A. Green, M. McLean, S.L. Olson, and J.J. Schirra (Warrendale, PA: TMS, 2000) 181-188.

13. M. Lamm and R.F. Singer, Metall and Mater Trans A, 38 (2007) 1177.

14. J.D. Miller and T.M. Pollock, Metall and Mater Trans A, 45 (2014) 411-425.
15. J.D. Miller, L. Yuan, P.D. Lee and T.M. Pollock, Acta Mater, 69 (2014) 47-59.

16. J.D. Miller and T.M. Pollock, Superalloys 2012, ed. E.S. Huron, R.C. Reed, M.C. Hardy, M.J. Mills, R.E. Montero, P.D Portella and J. Telesman (Warrendale, PA: TMS, 2012) 653662.

17. J.D. Miller and T.M. Pollock, Acta Mater, 78 (2014) 23-36.

18. W. Wang, A. Kermanpur, P.D. Lee and M. McLean, J Mater Sci, 38 (2003) 4385-4391.

19. Yang XL, Dong HB, Wang W, Lee PD. Mat Sci Eng A, 2004; 386:129-139.

20. N. D'Souza, M. Newell, K. Devendra, P.A. Jennings, M.G. Ardakani and B.A. Shollock, Mat Sci Eng A, 413-414 (2005) 567-570.

21. R.N. Grugel and Y. Zhou, Metall Mater Trans A, 20 (1989) 969-973.

22. C. Beckermann, J.P. Gu, W.J. Boettinger, Metall Trans A, 31 (2000) 2545-2557.

23. S. Tin and T.M. Pollock, J Mat Sci, 39 (2004) 7199-7205.

24. X. Xue and L. Xu, Mat Sci Eng A, 499 (2009) 69-73.

25. Ch.-A. Gandin, M. Eshelman and R. Trivedi, Metall and Mater Trans A, 27 (9) (1996) 2727-2737.

26. U. Paul and P.R. Sahm, Mat Sci Eng A, 173 (1993) 49-54.

27. S. Preibisch, S. Saalfeld, P. Tomancak, Bioinformatics, 25 (2009) 1463.

28. J.D. Miller, "Heat Extraction and Dendritic Growth during Directional Solidification of Single-Crystal Nickel-base Superalloys" (Ph.D. thesis, 2011)

29. T.M. Pollock and W.H. Murphy, Metall Trans A, 27 (1996) 1081-1094

30. W. Kurz and D.J. Fisher, Acta Metall, 29 (1) (1981) 11-20.

31. J.D. Hunt, Solidification and Casting of Metals, ed. J.D. Hunt (London: The Metals Society, 1979), 3-9. 